\title{
Hausdorff distance-based multiresolution maps applied to image similarity measure
}

\author{
E. Baudrier*a , G. Millon ${ }^{\text {b }}$, F. Nicolier ${ }^{\text {b }}$, R. Seulin ${ }^{\mathrm{c}}$ and S. Ruan ${ }^{\mathrm{b}}$

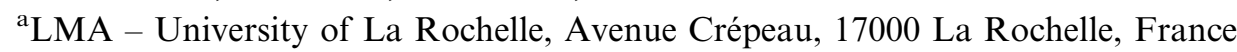 \\ ${ }^{\mathrm{b}}$ CReSTIC - URCA, IUT, 9, rue de Québec, 10026 Troyes Cedex, France \\ ${ }^{\mathrm{c}}$ Le2i - CNRS UMR 5158, University of Burgundy - IUT, 12, rue de la fonderie, 71200 Le Creusot, \\ France
}

\begin{abstract}
Image comparison is widely used in image processing. For binary images that are not composed of a single shape, a local comparison can be interesting because the features are usually poor (colour) or difficult to extract (texture, forms). Thus a new binary image comparison method that uses a windowed Hausdorff distance is presented. It enables local dissimilarities to be quantified in a simple way. The comparison results in a dissimilarity map. These maps are then used to evaluate the image similarity. The evaluation uses a classification step that is based on a comparison of the dissimilarity map histogram with reference histograms. The comparison is carried out at different scales of a multiresolution analysis, allowing the most discriminating scale in a user-defined notion of dissimilarity to be chosen automatically in the learning step. As an application, a database of digitalized ancient illustrations is successfully processed by the new method.
\end{abstract}

Keywords: image comparison, binary images, dissimilarity map, multiresolution, mathematical morphology

\section{INTRODUCTION}

Image retrieval is an active domain. Retrieving images by their content, as opposed to metadata, has become an important activity. It is classically composed of two stages: first, information mining, which results in an image signature and, secondly, a signature distance measure which is used to decide on the image similarity. In this process, the signature must capture conspicuous features in order to be as discriminating as possible in a user-defined sense. In general, the signature contains colour, shape or texture information. ${ }^{1}$ For the binary images that are not composed of a single shape, the colour attributes are poor. Often object shapes cannot be clearly

The MS was accepted for publication on 30 March 2006.

* Corresponding author: E. Baudrier, LMA - University of La Rochelle, Avenue Crépeau, 17000 La Rochelle, France; e-mail: etienne.baudrier@univ-lr.fr identified. In the scope of binary images, it is proposed to replace this information mining by a straight image comparison based on a modified Hausdorff distance (HD), ${ }^{2}$ producing a dissimilarity map (DM). The second step is then replaced by a classification process based on the DM. While information mining requires a priori knowledge on discriminating features before comparing the images, this process first expresses dissimilarities from the image comparison before taking a decision. This process developed for binary images can be adaptable to pattern recognition. In the present paper, the different stages of the measuring process are presented: first, a morphological multiresolution analysis (MRA) (section 2) and, secondly, the construction of DM between the two images (section 3) at each scale. Then, the choice of the most discriminating MRA scale and a decision on the similarity of the images based on the DM classification at this scale is 
presented at the end of section 5. Finally, some results are presented in section 6 to show the efficiency of this method.

\section{MULTIRESOLUTION}

The dissimilarity-measure definition depends on the database considered and on the degree of similarity chosen by the user (coarse or fine) at the same time. These parameters are taken into account thanks to a MRA during the learning step. This step determines first the best discriminating MRA scale for the classification and then the class reference histograms at this scale. This section gives a general presentation of the MRA, and then the chosen non-linear MRA filter is introduced.

\subsection{MRA for multidimensional signals}

The MRA is directly transposed from the 1D case described by Meyer ${ }^{3}$ and Mallat. ${ }^{4}$ In this paragraph, the properties and definitions are valid whatever the space dimension of the given functions (2D, 3D and so on). The processed functions are square-integrable over $R^{\mathrm{N}}$, so the signal $f$ belongs to $\boldsymbol{L}^{2}\left(R^{\mathrm{N}}\right)(N=2$ for $2 \mathrm{D}$ signals). A MRA is a sequence of successive approximations spaces $V_{\mathrm{j}}$ satisfying the following properties:

$$
\begin{aligned}
& \forall j \in Z, V_{\mathrm{j}} \subset V_{\mathrm{j}-1} \text { with } \bigcup_{\mathrm{j} \in Z} V_{\mathrm{j}}=L^{2}\left(R^{\mathrm{N}}\right) \text { and } \\
& \bigcap_{\mathrm{j} \in Z} V_{\mathrm{j}}=\{0\} \\
& f(x) \in V_{\mathrm{j}} \Leftrightarrow f\left(J^{\mathrm{j}} x\right) \in V_{0}, \forall j \in Z \\
& f(x) \in V_{0} \Rightarrow f(x-k) \in V_{0}, \forall k \in Z^{\mathrm{N}} \\
& \exists \varphi \in V_{0}, \text { so that }\left\{\varphi_{0, \mathrm{k}}, k \in Z\right\} \text { is an orthonormal } \\
& \text { basis of } \mathrm{V}_{0}
\end{aligned}
$$

where $\varphi_{\mathrm{j}, \mathrm{k}}(x)=|\operatorname{det} J|^{-\mathrm{j} / 2} \varphi\left(J^{-\mathrm{j}} x-k\right), \forall j Z, \forall k Z^{\mathrm{N}}$

where $J$ is defined by

$$
\left(\begin{array}{l}
x^{\prime} \\
y^{\prime}
\end{array}\right)=J\left(\begin{array}{l}
x \\
y
\end{array}\right)
$$

$J$ is an $N \times N$ matrix $(2 \times 2$ in $2 \mathrm{D})$ with integer coefficients (also for $|\operatorname{det} J| \cdot J^{-1}$ ). The scaling factor is $|\operatorname{det} J|$.

One can show that a base $W_{\mathrm{j}}$ is given by translations of $|\operatorname{det} J|-1$ families of wavelets functions. So for one given scale, there are $|\operatorname{det} J|-1$ detail signals $d_{\mathrm{j}, \mathrm{i}}$, and one approximation signal $a_{\mathrm{j}}$.
The multidimensional Mallat's recursive algorithm can be generalized without major modifications:

$$
\begin{aligned}
& a_{\mathrm{j}}=\bar{h} * a_{\mathrm{j}-1} \\
& d_{\mathrm{j}, \mathrm{k}}=\bar{g}_{\mathrm{k}} * a_{\mathrm{j}-1}
\end{aligned}
$$

where $\overline{g_{\mathrm{k}}}, \bar{h}$ are multidimensional kernels filters and * denotes the convolution operator. $\bar{h}$ is a low-pass filter, and $\overline{g_{\mathrm{k}}}$ are high-pass filters.

\subsection{Non-linear MRA}

An extension of the MRA to the non-linear case is presented here. Indeed, in the present study, the MRA filter should satisfy conditions to preserve the binary image main features. Many classical MRA filters use Gaussian functions. They have good space-scale properties, but they smooth transitions and could yield to new structures at low resolutions. This drawback is shared by the other linear filters. In contrast, non-linear filters can avoid this problem. Among them, morphological filters are good candidates. ${ }^{5}$ Here, three criteria have been determined for the morphological MRA filter $\tau$ choice: $\tau$ should be edge-preserving, $\tau$ should be auto-dual (i.e. $\tau$ preserves the black-to-white pixels ratio), and $\tau$ should preserve the 'main' features. Obviously, the last criterion is subjective and is satisfied a posteriori. This led to the choice of the morphological so-called median filter, which fulfils these conditions. ${ }^{6}$ The morphological MRA is thus described by the following process.

1. Non-linear median filtering of the approximation $a_{\mathrm{j}}$

2. Down-sampling by a factor 2 , giving $a_{\mathrm{j}-1}$

3. Repeat the process up to scale $J$.

Let I be the image to treat; the analysis filter results in an approximation image $\mathbf{A}$ and three detail images $\mathbf{D}_{\mathbf{v}}, \mathbf{D}_{\mathbf{d}}, \mathbf{D}_{\mathbf{h}}$ corresponding to the vertical, diagonal and horizontal details. The coefficients $\mathbf{A}(k, l), \mathbf{D}_{\mathbf{v}}(k, l)$, $\mathbf{D}_{\mathbf{d}}(k, l), \mathbf{D}_{\mathbf{h}}(k, l)$ are computed with the following operations:

$$
\begin{aligned}
& \mathbf{b}=\operatorname{sort}[\boldsymbol{I}(2 i-1,2 j-1), \boldsymbol{I}(2 i-1,2 j-1), \\
& \boldsymbol{I}(i-1, j), \boldsymbol{I}(i, 2 j-1), \boldsymbol{I}(2 i, 2 j)] \\
& \mathbf{A}(k, l)=\mathbf{b}(3) \\
& \boldsymbol{D}_{\mathbf{v}}(k, l)=|\boldsymbol{I}(2 i-1,2 j-1)-\boldsymbol{I}(2 i-1,2 j)| \\
& \boldsymbol{D}_{\mathbf{d}}(k, l)=|\boldsymbol{I}(2 i-1,2 j-1)-\boldsymbol{I}(2 i, 2 j)| \\
& \boldsymbol{D}_{\mathbf{h}}(k, l)=|\boldsymbol{I}(2 i-1,2 j-1)-\boldsymbol{I}(2 i, 2 j-1)|
\end{aligned}
$$

Two illustrations of the non-linear median filter are 

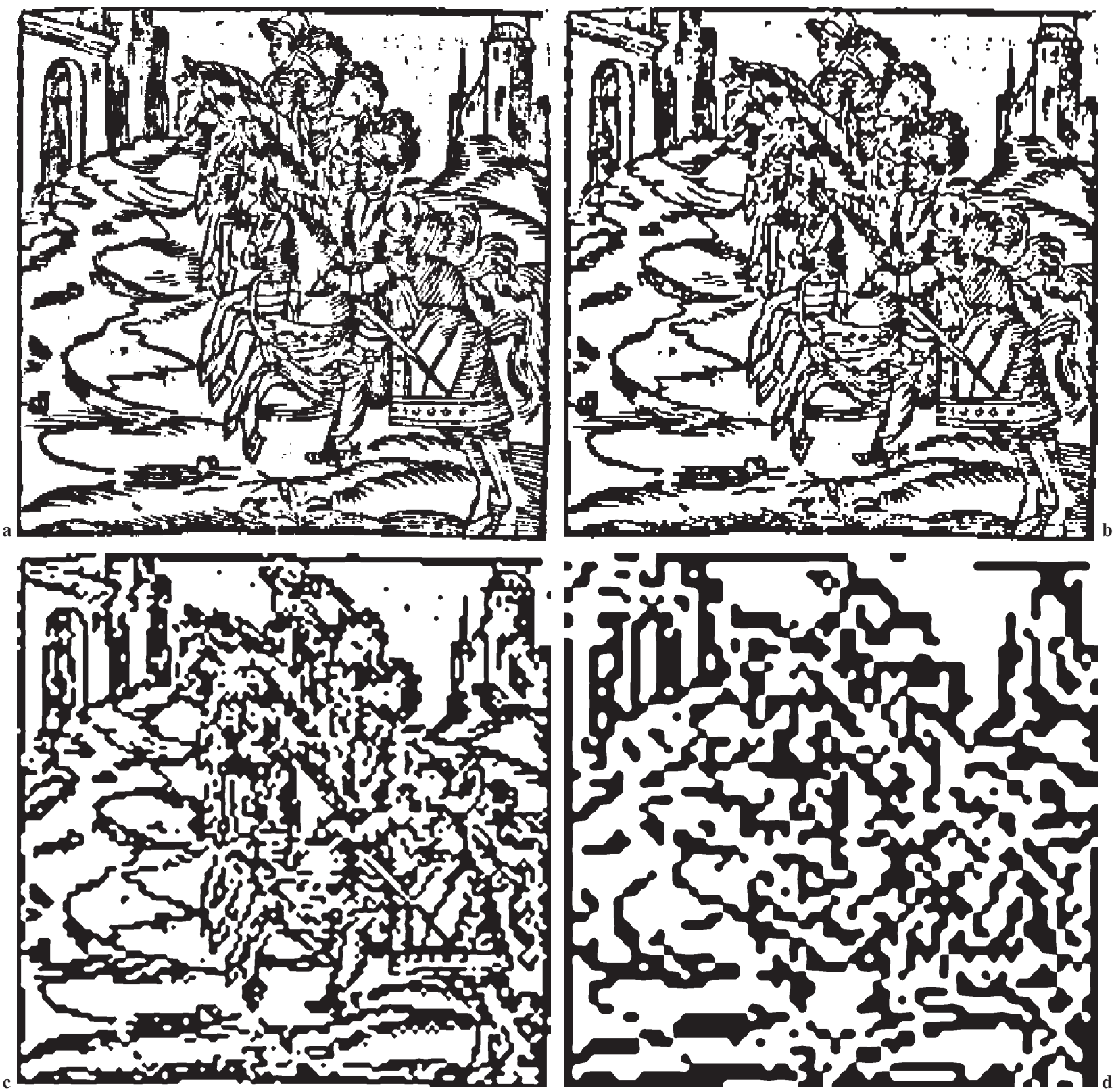

1 Four resolutions of same picture illustrating effect of non-linear median filter ((a) is original image with all detail; (d) keeps only the image main features): (a) binarized original image at resolution $0 \quad(512 \times 512$ pixels $)$; (b) resolution $1 \quad(256 \times 256$ pixels $)$; (c) resolution 2 $(128 \times 128$ pixels $) ;(d)$ : resolution $2(64 \times 64$ pixels $)$

presented Figs 1 and 2. Figure 1 represents the same image at four successive scales, from the finest (Fig. 1a) to the coarsest (Fig. 1d). In the coarsest one, the image quality is degraded; nevertheless the main features are still visible. Figure 2 stands for the first approximation of the same image and the three detailed images at scale 1 (the detail images will not be used afterwards).

\section{OVERVIEW OF HAUSDORFF DISTANCE}

3.1 Dissimilarity measure over binary images: choice of Hausdorff distance

Among dissimilarity measures over binary images, the HD has often been used in the content-based retrieval domain and is known to have successful applications in object matching ${ }^{2,7}$ or in face 

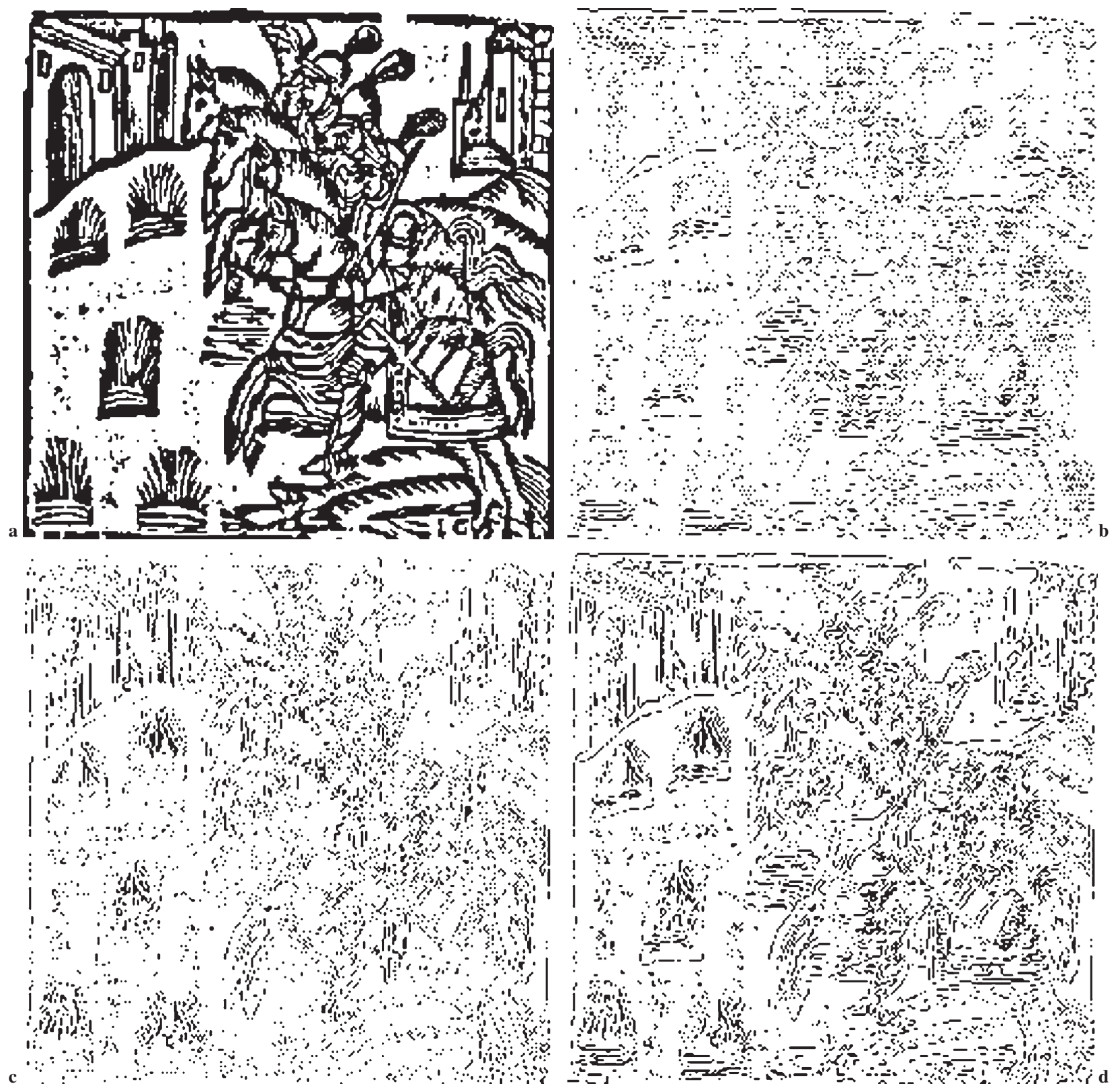

2 Result of non-linear median AMR: approximation 1 and corresponding three detail images: (a) resolution $1(256 \times 256$ pixels); (b) vertical detail; (c) horizontal detail; (d) diagonal detail

recognition. ${ }^{8,9}$ It can be computed quickly using Voronoi diagrams. ${ }^{10}$ A brief review of its definition and properties follows.

Originally meant as a measure between two point collections $A$ and $B$ in a metric space $E$ (whose underlying distance is $d$ ), it can be viewed as a dissimilarity measure between two binary images $\mathbf{A}$ and $\mathbf{B}$, considering $A$ and $B$, respectively, the black pixels of $\mathbf{A}$ and $\mathbf{B}$. For finite sets of points, the HD can be defined as: ${ }^{11}$

Definition 1. The Hausdorff distance

Given two finite sets of points $\mathbf{A}=\left(a_{1}, \ldots, a_{\mathrm{n}}\right)$ and $\mathbf{B}=\left(b_{1}, \ldots, b_{\mathrm{m}}\right)$, and an underlying distance $d$, the HD is given by

$$
D_{\mathrm{H}}(\mathbf{A}, \mathbf{B})=\max [\mathrm{h}(\mathbf{A}, \mathbf{B}), \mathrm{h}(\mathbf{B}, \mathbf{A})]
$$

where

$$
\mathrm{h}(\mathbf{A}, \mathbf{B})=\max _{\mathrm{a} \in \mathrm{A}}\left\{\min _{\mathrm{b} \in \mathbf{B}}[d(a, b)]\right\}
$$

$\mathrm{h}(\mathbf{A}, \mathbf{B})$ is the so-called 'directed HD' and, in the present paper, the underlying distance $d(a, b)$ will be the $L_{\infty}$ norm $d(a, b)=\|b-a\|_{\infty}$. For images, the same notation is used: $D_{\mathrm{H}}(\mathrm{A}, \mathrm{B})=D_{\mathrm{H}}(\mathbf{A}, \mathbf{B})$. The interest of this measure comes first from its metric properties (in this application, on the space of finite set of points): non-negativity, identity, symmetry and triangle 
inequality. These properties correspond to this intuition for shape resemblance. Indeed, a pattern is identical to itself. And the order of comparison does not generally matter; for the case where the order of comparison is important, the property of the HD according to which the directed distance is not symmetrical can be exploited. Finally, the triangular inequality prevents some unknown patterns being similar to two dissimilar patterns at the same time.

Moreover, the HD is a match methodology without point-to-point correspondence, so it is robust to local non-rigid distortions. Another source of interest is the following property.

Proposition 1 (Translation): Let $\mathbf{v}$ be a vector of $R^{2}$, $T_{\mathbf{v}}$ translation of vector $\mathbf{v}$, and $A$ a non-empty finite set of points, then

$$
d\left(A, T_{v} A\right)=\|\mathbf{v}\|
$$

It implies that for a small translation, the HD is small, which matches the expectation for a dissimilarity measure.

\subsection{Some modified versions of the HD}

The classical HD has good properties but it measures the most mismatched points between $A$ and $B$ and, as a consequence, it is sensitive to noise. ${ }^{12}$ Indeed considering two images containing the same pattern and one point added to the first image far from the pattern, the HD will measure the distance between the pattern and the point.

Several modifications of the HD have been proposed to improve it, such as the partial $\mathrm{HD},{ }^{11}$ the modified HD (MHD), ${ }^{13}$ the censored HD (CHD) ${ }^{12}$ the 'doubly' modified HD (M2HD), ${ }^{8}$ the least trimmed squared HD (LTS-HD) ${ }^{14}$ and the weighted HD (WHD). ${ }^{15}$ The next definitions are detailed by Zhao et al. ${ }^{16}$

The directed distance of the partial HD is defined by Huttenlocher et al.: ${ }^{11}$

$$
h_{\mathrm{K}}(A, B)=K_{\mathrm{a} \in \mathrm{A}}^{\mathrm{th}} d(a, B)
$$

where $K_{\mathrm{a} \in \mathrm{A}}^{\mathrm{th}}$ denotes the $K$ th ranked value of $d(a, B)$. Thus, the PHD depends on a parameter $p=\frac{K}{N_{A}}$ standing for the proportion of values taken into account. The partial HD method yields good results for an impulse noise case.

The directed distance of the MHD is defined by Dubuisson and Jain: ${ }^{13}$

$$
h_{\mathrm{MHD}}(A, b)=\frac{1}{N_{\mathrm{A}}} \sum_{\mathrm{a} \in \mathrm{A}} d(a, B)
$$

where $N_{\mathrm{A}}=\operatorname{card}(A)$. Unlike the partial HD, the MHD measure does not require any parameter, and the method adapts only to a Gaussian noise case.

The directed distance of the WHD is defined by Zhao et al.: ${ }^{16}$

$$
h_{\mathrm{WHD}}(A, B)=\frac{1}{N_{\mathrm{A}}} \sum_{\mathrm{a} \in \mathrm{A}} \omega(a) \cdot d(a)
$$

where $\sum_{\mathrm{a} \in \mathrm{A}} \omega(a)=N_{\mathrm{A}}$. An image can be divided into different parts, and the contribution of the different parts to the image matching is different, therefore the HD should be different. The WHD has been used in the Chinese character image matching ${ }^{15,17}$ and in face recognition. ${ }^{18}$

The directed distance of the CHD is defined by Paumard: ${ }^{12}$

$$
h_{\mathrm{k}, 1}(A, B)=P_{\mathrm{a} \in \mathrm{A}}^{\mathrm{th}} Q_{\mathrm{b} \in \mathrm{B}}^{\mathrm{th}} d(a, b)
$$

where $P^{\text {th }}$ denotes the $P$ th ranked value for $a A$ of $Q_{\mathrm{b} \in \mathrm{B}}^{\text {th }} d(a, b)$, with $Q_{\mathrm{b} \in \mathrm{B}}^{\text {th }} d(a, b)$ representing the $Q$ th ranked value for $b B$ of the underlying distance set. Since the CHD ranks the underlying distance, the effect of the impulse noise to the image is reduced.

The directed distance of the M2HD is defined by Takàcs: ${ }^{8}$

$$
h_{\mathrm{M}}=\frac{1}{N_{\mathrm{A}}} \sum_{\mathrm{a} \in \mathrm{A}} d(a, B)
$$

with

$$
d(a, B)=\max \left(I \cdot \min _{\mathrm{b} \in \mathrm{N}_{\mathrm{B}}^{\mathrm{a}}} d(a, b),(1-I) P\right)
$$

where $N_{\mathrm{B}}^{\mathrm{a}}$ is a neighbourhood of point $a$ in set $B$, and $I$ indicates whether a point $b$ exists in $N_{\mathrm{B}}^{\mathrm{a}}$.

The directed distance of the LTS-HD is defined by Sim et al.: $:^{14}$

$$
h_{\mathrm{LTS}}(A, B)=\frac{1}{K} \sum_{1}^{\mathrm{K}} d(a, B)_{\mathrm{(i}}
$$

where $K$ denotes $k \cdot N_{\mathrm{A}}$, as in the partial HD case, and $d(a, B)_{(\mathrm{i})}$ represents the $i$ th distance value in the sorted sequence $d(a, B)_{(1)} \leqslant d(a, B)_{(2)} \leqslant \ldots \leqslant d(a, B)_{\left(\mathrm{N}_{\mathrm{A}}\right)}$. The measure of the LTS-HD is minimized by the remaining distance values, after large distance values are eliminated. Even if the object is occluded or degraded by noise, this matching scheme yields good results.

These measures are global and cannot account for local dissimilarities. Indeed, the principle of HD is to be a 'max min' distance, and this means that the value of the HD between two images is reached for at least a couple of points. But this does not say whether the 


\section{Discrete case}

\section{$\square$ Points of A $\square$ Points of B}

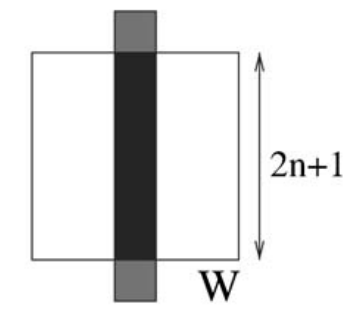

Naive HD non defined

Distance to $\operatorname{Fr}(\mathrm{W})=\mathrm{n}$

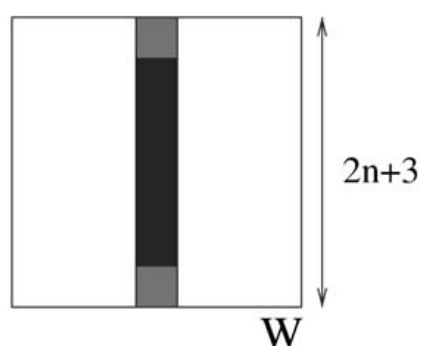

Naive $\mathrm{HD}=\mathrm{n}+1$

Distance to $\operatorname{Fr}(\mathrm{W})=\mathrm{n}+1$

(a)
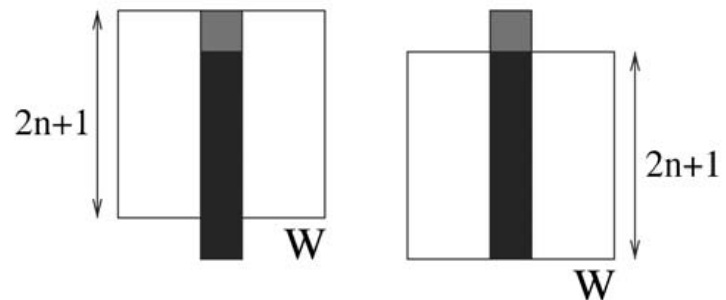

Modified local HD $=2 \mathrm{n}$ Modified local HD $=\mathrm{n}$

Final local $\mathrm{HD}=\mathrm{n} 1 \quad$ Final local $\mathrm{HD}=\mathrm{n}$

(b)

3 Examples of critical cases for local HD: (a) $B \cap W=\varnothing$, naive measure is not defined; (b) to define $H D_{\mathrm{W}}$ and to make it consistent where $W$ increases, distance to frontier is proposed; (c, d) definition must then be modified to be consistent when $W$ slides in $R^{2}$.

value is reached in several parts or only for one pair, which corresponds to different degrees of dissimilarity. These remarks motivate us to design a local HD in the next section.

\section{HAUSDORFF DM}

The main inconvenience of these HD measures lies in the fact that they give a global dissimilarity measure over images. To retrieve different images standing for the same scene, the image comparison must be based on the measure of local differences. To do so, a new dissimilarity measure based on the HD is introduced and designated $D_{\mathrm{H}, \mathrm{W}}$. It consists in making a local measure through a sliding window $W$. At each sample point, the HD is computed on the part of the images viewed through $W$. Thus, from two binary images to be compared, a DM $M$ is computed. $M$ depends on the parameters $\left(w_{\mathrm{x}}, w_{\mathrm{y}}\right)$, the size of the window $W$ and $p=\left(p_{\mathrm{h}}, p_{\mathrm{v}}\right)$, the step between the sample points.

\subsection{Windowed HD}

In this section, the notion of local dissimilarity is first discussed, then a naive definition of a HD measure in a local window is presented. The use of the HD in a local window implies some modifications to make it consistent when window size varies. A necessary modification is brought. It finally leads to a fully consistent definition. In this section, $A$ and $B$ depict two non-empty finite sets of points of $R^{2}$, and $W$ a convex closed subset of $R^{2}$.

\subsubsection{Naive definition}

This consists in modifying the definition of the global measure (Definition 1) by introducing a subset $W$ standing for the window.

Definition 2: $H D$ in a window (naive)

$$
H D_{\mathrm{W}}(A, B)=\max \left(h_{\mathrm{W}}(A, B), h_{\mathrm{W}}(B, A)\right)
$$

where

$$
h_{\mathrm{W}}(A, B)=\max _{\mathrm{a} \in \mathrm{A} \cap \mathrm{W}}\left\{\min _{\mathrm{b} \in \mathrm{B} \cap \mathrm{W}}[d(a, b)]\right\}
$$

But this definition is not available for the possible case where one set (for example $B$ ) has no point in $W$, and the other one has. So as to make the value consistent when $W$ grows (see Fig. 3a,b), the distance to the frontier of $W$ must be taken into account.

\subsubsection{Modification of the naive definition}

Indeed, if there are points of $A$ outside $W$ and close to its frontier (Fig. 3a), if $W$ grows and includes them, the new $H D_{\mathrm{W}}$ will be equal to the distance to these points. The most restrictive case will be when there are points of $A$ all over the frontier of $W$. Then, to make it consistent with a bigger $W$, the computation of $H D_{\mathrm{W}}$ must include the distance of the points of the two sets $A$ and $B$ to the edges of $W$. Thus, if $B$ has no point in $W$, the directed distances have the following definition: 
Definition 2: First modification

$$
H D_{\mathrm{W}}(A, B)=\max \left(h_{\mathrm{W}}(A, B), h_{\mathrm{W}}(B, A)\right)
$$

where there are two cases:

$$
\begin{aligned}
& \text { If } A \cap W \neq \varnothing \text { and } B \cap W \neq \varnothing \\
& h_{\mathrm{W}}(A, B)=\max _{\mathrm{a} \in \mathrm{A} \cap \mathrm{W}}\left[\min _{\mathrm{b} \in \mathrm{B} \cap \mathrm{W}} d(a, b)\right]
\end{aligned}
$$

If $A \cap W \neq \varnothing$ and $B \cap W=\varnothing$

$$
h_{\mathrm{W}}(A, B)=\max _{\mathrm{a} \in \mathrm{A} \cap \mathrm{W}}\left[\min _{\mathrm{w} \in \mathrm{Fr}(\mathrm{W})} d(a, w)\right]
$$

If $A \cap W=\varnothing$

$$
h_{\mathrm{W}}(A, B)=0
$$

The frontier $\operatorname{Fr}(W)$ comes from the topology defined by the metric $d$. In the application (section 6), the distance is the one associated to the norm $L_{\infty}$.

\subsubsection{Local HD}

Once this case is clarified, the definition in the global case must be modified to bring a consistency when the window slides over the images. Indeed, taking two cases in the definition will bring sharp variations of the distance value when the window slides and would not reflect the intuition (see Fig. 3b). Then the distance to the frontier of $W$ has to be taken into account in the definition.

Definition 2. Final version: the $H D$ in a window

Let $A$ and B be two finites sets of $R^{2}$

$$
H D_{\mathrm{W}}(A, B)=\max \left(h_{\mathrm{W}}(A, B), h_{\mathrm{W}}(B, A)\right)
$$

where there are three cases:

$$
\begin{aligned}
& \text { If } A \cap W \neq \varnothing \text { and } B \cap W \neq \varnothing \\
& h_{\mathrm{W}}(A, B)=\max _{\mathrm{a} \in \mathrm{A} \cap \mathrm{W}}\left[\min _{\mathrm{b} \in \mathrm{B} \cap \mathrm{W}} d(a, b), \min _{\mathrm{w} \in \mathrm{Fr}(\mathrm{W})} d(a, w)\right]
\end{aligned}
$$

If $A \cap W \neq \varnothing$ and $B \cap W=\varnothing$

$$
h_{\mathrm{W}}(A, B)=\max _{\mathrm{a} \in \mathrm{A} \cap \mathrm{W}}\left[\min _{\mathrm{w} \in \mathrm{Fr}(\mathrm{W})} d(a, w)\right]
$$

If $A \cap W=\varnothing$

$$
h_{\mathrm{W}}(A, B)=0
$$

\subsection{Properties}

$H D_{\mathrm{W}}$ has the following properties:

$$
\begin{aligned}
& H D_{\mathrm{W}}(A, B)=H D_{\mathrm{W}}(B, A) \text { (Symmetry) } \\
& H D_{\mathrm{W}}(A, B)=[0] \Leftrightarrow A=B \text { (Identity) }
\end{aligned}
$$

These properties come from the metric properties of the HD. In contrast, the triangular inequality is no more verified.

$$
\text { If }\left(w_{\mathrm{x}}, w_{\mathrm{y}}\right)=(1,1) \text { and } p=1, H D_{\mathrm{W}}(A, B)=|B-A|
$$

Property 25 means that, with the smallest window size and the smallest step, the DM is equal the pixelto-pixel difference between $A$ and $B$.

$$
\begin{aligned}
& \text { If }\left(w_{\mathrm{x}}, w_{\mathrm{y}}\right)=(2 m, 2 n), \\
& \text { then } H D_{\mathrm{W}}(A, B)=H D(A, B)
\end{aligned}
$$

Here, this means that all the measures have the same value (equal to the global HD) in the DM, when the window includes the images.

\subsection{Examples of DM}

Figure 4 gives some examples of DM between image 1 and images 2, 3 and 4. Images 1 and 2 are very similar, and they result in a DM with low distance values. Image 3 illustrates the same scene as image 1, but the grass and the helms have been represented differently. In their DM, the highest values are confined to these parts. Image 3 is completely different from image 1 and, in their DM, high values are more numerous.

It is possible to take different sizes for the sliding window. For a given resolution, the larger the sliding window, the coarser the measured dissimilarities. An illustration is given in Fig 5.

Thus, a variation in the window size enables different sizes of dissimilarities to be highlighted. As the human comparison process is a coarse-to-fine process, it is interesting to begin the comparison with a large sliding window. Nevertheless, it is time consuming. Another way of using this property is to keep the same sliding window at different resolutions.

\section{CLASSIFICATION}

\subsection{DM classification}

At a fixed scale, the comparison process results in a DM. It can be classified in two ways: $C_{\text {sim }}$, which includes the DM comparing similar images, and $C_{\text {dissim }}$ for those comparing dissimilar images. The classification is based on the comparison of the DM histogram to class reference histograms. The reference histograms are the average histograms over each class. The DM histogram is then compared with the reference histogram, using a nearest-neighbour method with a Euclidean distance. Thus, a decision is taken at each scale, the next paragraph presents the contribution of the MRA to the decision process. 

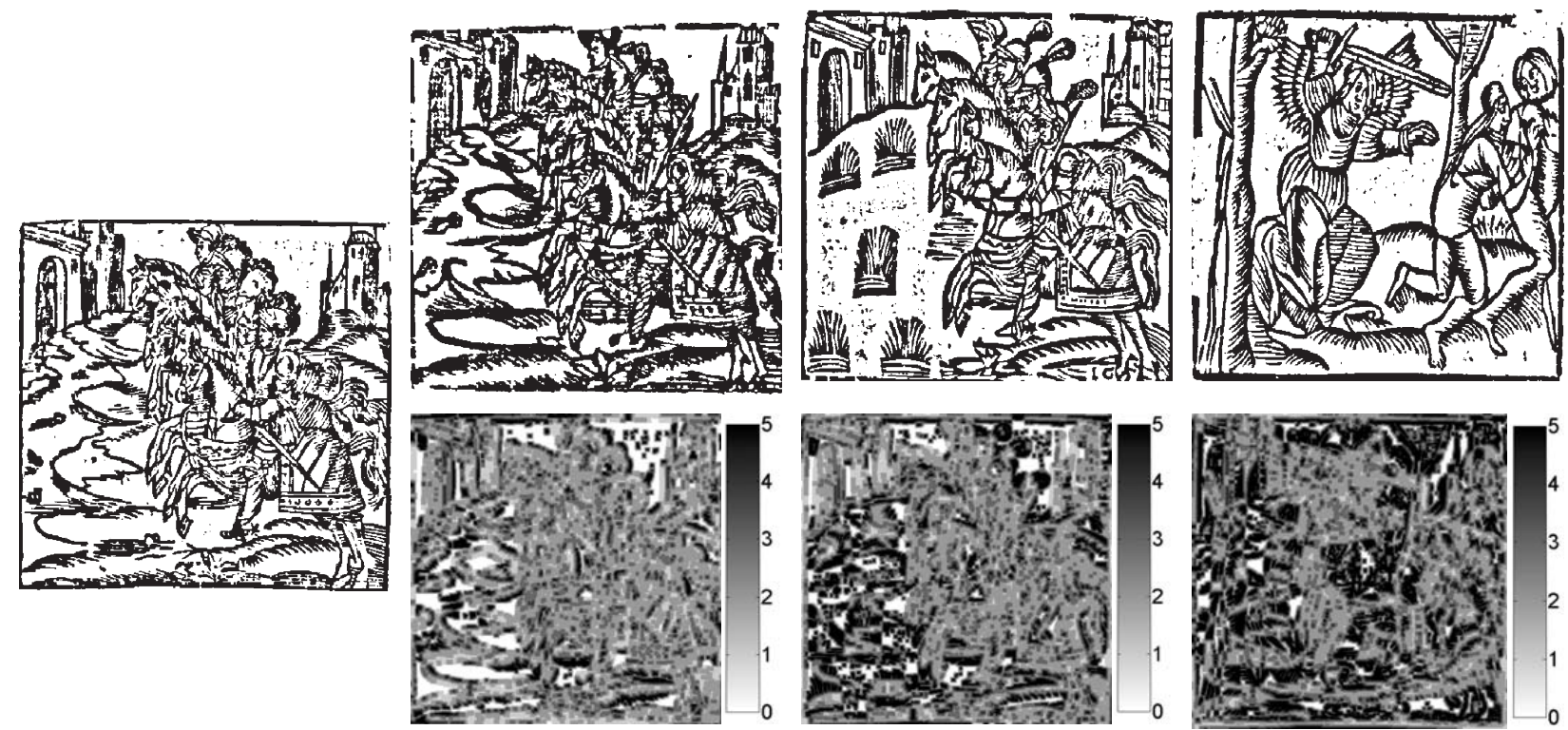

4 Some pictures and their distance maps

\subsection{Multiresolution and decision}

\subsubsection{Multiresolution and image quality}

As the MRA is used in the classification process, it must be shown that the MRA filter does not distort the images too much from a classification point of view. For this purpose, DM have been computed at different scales, with a sliding window proportional to the scale (so as to highlight the same kind of dissimilarities). The classification (presented in the next section) must then be applied to the DM to evaluate the classification rates at each scales. The results are presented in Table 1, which contains the results of 100 comparisons done at resolution 1 (with the original images), resolution 4 (size divided by 8 ) and resolution 6 (size divided by 32). It shows that the MRA does not affect (to some extent) the classification: the results are obviously worse at resolution 6 ,
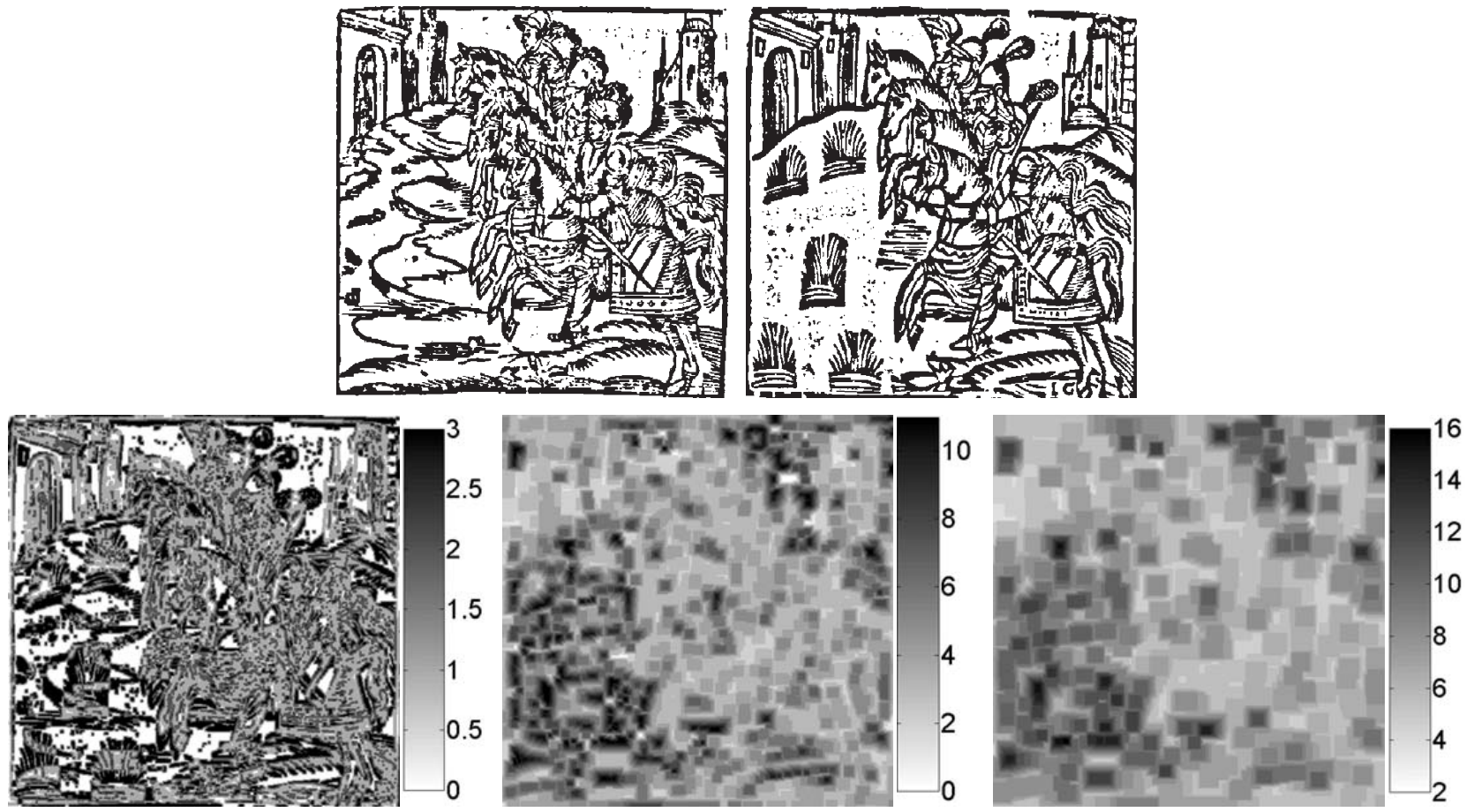

$55 \times 5$ sliding window shows fine dissimilarities, unlike $35 \times 35$ sliding window which overtones coarse ones (grass and helms) 


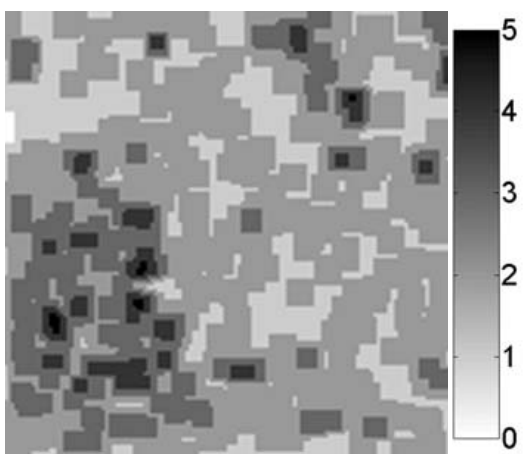

a

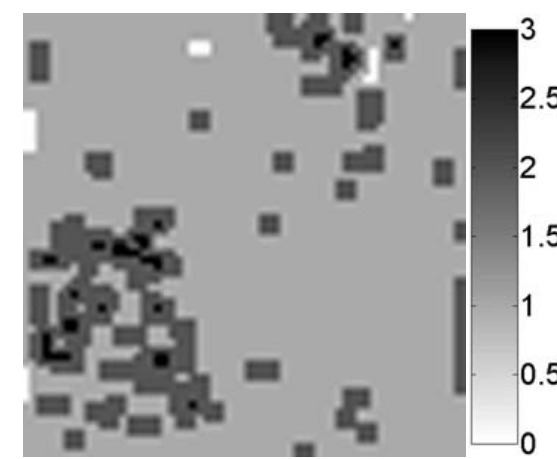

b

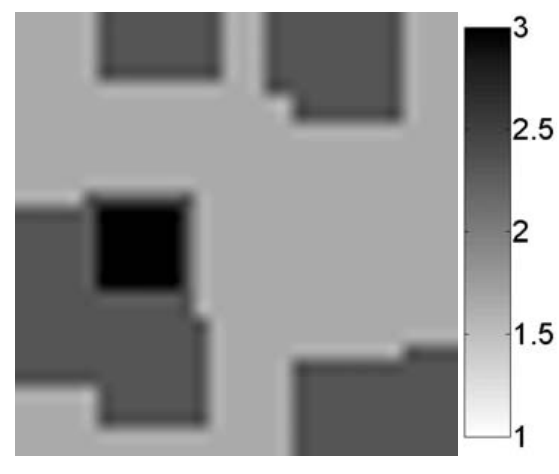

c

6 Distance maps of images 1 and 2. Resolution 2 shows dissimilarities even in the riders and their mounts; only the coarse dissimilarities (grass and helms) remain in resolution 4: (a) distance map at resolution $2(128 \times 128), 10 \times 10$ sliding window; (b) distance map at resolution $3(64 \times 64)$, $10 \times 10$ sliding window; (c) distance map at resolution $4(32 \times 32), 10 \times 10$ sliding window

because information is damaged; nevertheless, the classification remains quite right. For the present database, resolution 4 offers better results, a property that will be exploited in the decision process.

\subsubsection{Best scale choice for the classification}

Let $I$ and $J$ be two images $2 m \times 2 n, \tau(I)$ and $\tau(J)$ their $m \times n$ approximations produced with the MRA operator $\tau$. The sliding window size is kept the same for both of the resolutions to produce the DM. If the sliding window size is constant across scales, thus a high-resolution DM highlights fine dissimilarities and a coarse-resolution one highlights coarse dissimilarities. Figure 6 illustrates this property. Indeed, it shows the DM of the same images at three different scales, with a constant sliding window size equal to $10 \times 10$. The pieces of detail highlighted in Fig. $6 \mathrm{a}$ are finer than those in Fig. 6c. These DM are comparable to the ones computed at scale 1 with different sliding window sizes (Fig. 5). The dissimilarities highlighted are similar, but the computation at a coarse resolution with a fixed sliding window size is faster.
Thus, for the decision process, the classification rates are computed at each scale over the learning set. Then the scale with the best rate is selected for the global process (this choice depends on the database and on the leaning set). In Table 2, the classification rates at six scales are presented for the same learning set of the application database. The results lead to the choice of resolution 4 .

\section{RESULTS}

The proposed method is tested on the database of digitalized ancient illustrations provided by the Troyes' library within the framework of a collaboration with the CReSTIC. ${ }^{19}$ These images, originally printed on books with dark ink, have a strong contrast, which allows them to be binarized with almost no loss. This database is composed of 68 images, some of them illustrating the same scene. The objective is to retrieve illustrations of the same scene. First, 2380 DM were built, 103 of which are classified in $C_{\text {sim }}$ thanks to a manual comparison of the

Table 1 Results of classification of $100 \mathrm{DM}$ at resolution 1, 4 and 6 with sliding window size proportional to image size

\begin{tabular}{llll}
\hline Successful retrieval & Scale 1 & Scale 4 & Scale 6 \\
\hline Found in $C_{\text {sim }}$ & $72 \%$ & $84 \%$ & $62 \%$ \\
Found in $C_{\text {dissim }}$ & $86 \%$ & $95 \%$ & $72 \%$ \\
\hline
\end{tabular}

Table 2 Results of classification of learning set of 100 DM at resolution $1-6$ with $10 \times 10$ sliding window size

\begin{tabular}{lllllll}
\hline Successful retrieval & Resolution 1 & Resolution 2 & Resolution 3 & Resolution 4 & Resolution 5 & Resolution 6 \\
\hline Found in $C_{\text {sim }}$ & $78 \%$ & $78 \%$ & $76 \%$ & $82 \%$ & $70 \%$ & $62 \%$ \\
Found in $C_{\text {dissim }}$ & $92 \%$ & $86 \%$ & $94 \%$ & $94 \%$ & $90 \%$ & $72 \%$ \\
\hline
\end{tabular}


Table 3 Results for DM, global HD and for DM based on absolute difference measure

\begin{tabular}{llll}
\hline Successful retrieval & DM & HD & $S D_{\mathrm{W}}$ \\
\hline Found in $C_{\text {sim }}$ & $\mathbf{8 4} \%$ & $46 \%$ & $66 \%$ \\
Found in $C_{\text {dissim }}$ & $\mathbf{9 5} \%$ & $70 \%$ & $78 \%$ \\
\hline
\end{tabular}

impressions (and the others in $C_{\mathrm{dissim}}$ ). The objective is to test the method's efficiency in assessing local dissimilarities. The experiments was carried out by the following way: first a learning is made on a set of $50 \mathrm{DM}$ in $C_{\text {sim }}$ and $50 \mathrm{DM}$ in $C_{\text {dissim. }}$.

Then, the test is done on a distinct set of $50 \mathrm{DM}$ of $C_{\text {sim }}$ and 50 of $C_{\text {dissim. }}$. The choice of the sets in each class is randomized. Secondly, three classification methods are applied. Finally, the results obtained are compared manually and automatically. The three classification methods are the following:

- the method based on the DM

- the one based on the global HD instead of the DM

- the method based on the $S D_{\mathrm{W}}$ which uses also a DM, but with the simple difference as local measure instead of the $\mathrm{HD}: S D_{\mathrm{W}}(A, B)=$ sum $|A \cap W-B \cap W|$.

The results are summarized in Table 3. They show the efficiency of the DM both concerning spatial information (comparison with the global HD) and concerning the ability of the local HD to catch the local dissimilarities (comparison with the $S D_{\mathrm{W}}$ ).

\section{CONCLUSION}

In the present paper, a new image comparison method was presented, which preserves the measuring properties of the HD and suppresses one of its inconveniences, the outlier sensibility. At the same time, the DM quantifies the dissimilarities between the compared images. As a consequence, the DM produced has a different distribution, whether the images are similar or dissimilar. This dissimilarity measure has been embedded in an AMR process, which results in a coarse-to-fine process. The good results of this method illustrate the benefits of the DM. Moreover, it enables the final user to find the dissimilarity zones between the compared images at a glance. The perspectives of the study are to improve the distance map by making the size of the sliding window automatically chosen and adaptive, and then to use a decision method taking in the whole distance map, so as to exploit the spatial information in it. Finally, it is planned that the improved method be applied to different databases so as to test its efficiency and robustness.

\section{REFERENCES}

1 Smeulders, A. W. M., Worring, M., Santini, S., Gupta, A. and Jain, R. Content based image retrieval at the end of the early years. IEEE Trans. Pattern Anal. Mach. Intell., 2000, 22, 1349-1380.

2 Huttenlocher, D. P. and Rucklidge, W. J. A multiresolution technique for comparing images using the Hausdorff distance. IEEE Trans. Pattern Anal. Machine Intell., 1993, 705-706.

3 Meyer, Y. Ondelettes et opérateurs, 1990 (Hermann, Paris).

4 Mallat, S. A theory for multiresolution signal decomposition: the wavelet representation. IEEE Trans. Pattern Anal. Machine Intell., 1989, 11, 674-694.

5 Vachier, C. Morphological scale-space analysis and feature extraction, Proc. Int. Conf. on Image Processing (ICIP), October 2001.

6 Heijmans, H. J. A. M. and Goutsias, J. Multiresolution signal decomposition schemes. Part 2: Linear and morphological pyramids. IEEE Trans. Image Process., 2000, 9, 1897-1913.

7 Kwon, O.-K., Sim, D.-G. and Park, R.-H. Robust Hausdorff distance matching algorithms using pyramidal structures. Pattern Recognition, 2001, 34, 2005-2013.

8 Takàcs, B. Comparing faces using the modified Hausdorff distance. Pattern Recognition, 1998, 31, 1873-1881.

9 Jesorsky, O., Kirchberg, K. J. and Frischholz, R. W. Robust face detection using the Hausdorff distance. In Audio- and Video-based Person Authentification AVBPA 01 (Eds Bigun and Smeraldi), Halmstadt, Sweden, 2001, Lectures Notes in Computer Science, vol. 2091, pp. 90-95 (Springer, Berlin).

10 Borgefors, G. Distance transformations in digital images. Comp. Vision Graph. Image Process., 1986, 34, 344-371.

11 Huttenlocher, D. P., Klanderman, D. and Rucklidge, W. J. Comparing images using the Hausdorff distance, IEEE Trans. Pattern Anal. Machine Intell., 1993, 15, 850-863.

12 Paumard, J. Robust comparison of binary images. Pattern Recognition Lett., 1997, 18, 1057-1063.

13 Dubuisson, M.-P. and Jain, A. K. A modified Hausdorff distance for object matching, Proc. 12th Int. Conf. on Pattern Recognition (ICPR), Jerusalem, Israel, 1994, pp. 566-568.

14 Sim, D.-G., Kwon, O.-K. and Park, R.-H. Object matching algorithms using robust Hausdorff distance 
measures. IEEE Trans. Image Process., 1999, 8, 425429.

15 Lu, Y., Tan, C., Huang, W. and Fan, L. An approach to word image matching based on weighted Hausdorff distance, Proc. 6th Int. Conf. on Document Anal.

3 Recogn, September 2001, pp. 921-925.

16 Zhao, C., Shi, W. and Deng, Y. A new Hausdorff distance for image matching. Pattern Recognition Lett., 2004.

$17 \mathrm{Lu}$, Y. and Tan, C. L. Word spotting Chinese document images without layout analysis, Proc. 16th
Conf. on Pattern Recognition, August 2002, vol. 3, pp. 57-60.

18 Guo, B., Lam, K., Siu, W. and Yang, S. Human face recognition using a spatially weighted Hausdorff distance, IEEE Int. Symp. on Circuits and Systems, 2001, pp. 145-148.

19 Seulin, R., Morel, O., Millon, G. and Nicolier, F. Range image binarization: application to wooden stamps analysis, in Int. Conf. on Quality Control by Artificial Vision $(Q C A V)$ - IEEE, Gatlinburg, TN, May, Proc. SPIE, Vol. 5132, 2003, pp. 252-258 (SPIE, Bellingham, WA). 


\section{Authors Queries}

Journal: The Imaging Science Journal

Paper: mp062

Title: Hausdorff distance-based multiresolution maps applied to image similarity measure

Dear Author

During the preparation of your manuscript for publication, the questions listed below have arisen. Please attend to these matters and return this form with your proof. Many thanks for your assistance

\begin{tabular}{|l|l|l|}
\hline $\begin{array}{l}\text { Query } \\
\text { Reference }\end{array}$ & Remarks \\
\hline 1 & $\begin{array}{l}\text { Author: please give name and } \\
\text { location of publisher. }\end{array}$ & \\
\hline 2 & $\begin{array}{l}\text { Author: please give name and } \\
\text { location of publisher. }\end{array}$ & \\
\hline 3 & $\begin{array}{l}\text { Author: please give name and } \\
\text { location of publisher. }\end{array}$ & \\
\hline 4 & $\begin{array}{l}\text { Author: please give volume and } \\
\text { page numbers. }\end{array}$ & \\
\hline 5 & $\begin{array}{l}\text { Author: please give name and } \\
\text { location of publisher. }\end{array}$ & \\
\hline 6 & $\begin{array}{l}\text { Author: please give name and } \\
\text { location of publisher. }\end{array}$ & \\
\hline 7 & $\begin{array}{l}\text { Author: please confirm publisher } \\
\text { correct. }\end{array}$ & \\
\hline
\end{tabular}

